\title{
The Impact of Climate Change on Human Health
}

\author{
Mary Snow and Rich Snow* \\ Embry-Riddle Aeronautical University, USA
}

"Corresponding author: Rich Snow, Embry-Riddle Aeronautical University, USA, Tel: 386-226-7104; E-mail: snow4fc@erau.edu

Received date: Jan 26, 2015, Accepted date: Jan 27, 2015, Published date: Jan 29, 2015

Copyright: (c) 2015 Snow R et al. This is an open-access article distributed under the terms of the Creative Commons Attribution License, which permits unrestricted use, distribution, and reproduction in any medium, provided the original author and source are credited.

\section{Editorial}

Climate change is impacting human health. An obvious effect of a warmer environment is more frequent and severe heat waves. During the European heat wave of 2003, there were an estimated 35,000 more deaths than normal in the first two weeks of August. Many of the deaths resulted from cardiovascular complications among the elderly. As heat waves become more commonplace in the future, so will the number of heat strokes and the onset of other cardiovascular problems. Higher summertime temperatures also increase tropospheric ozone concentrations which in turn affects people with asthma and causes lung and heart damage. Increases in the levels of photochemical pollutants like ozone are associated with a warming world.

Habitat modification also results from climate change and alters the regional prevalence of certain vector-borne diseases. A warmer environment and the accompanying incidence of extreme weather events will continue to affect the strength and distribution of certain diseases that favor standing water. The history of humankind both past and present has shown us that heat waves and cold spells as well as floods and droughts are often accompanied by disease outbreaks. Respiratory infections and other diseases run rampant when people are forced into crowded shelters due to tropical storms or flooding. In the aftermath of extreme weather events, conditions tend to favor many of the insects, bacteria, viruses and protozoa that spread disease. Higher temperatures and rainfall amounts affect the life cycles of disease carrying insects such as mosquitoes and ticks. Changes in weather affect contagious diseases such as influenza and pneumonia, as well as allergic diseases such as asthma while higher levels of humidity increase the incidence and severity of fungal infections and infestations.

For example, while West Nile encephalitis virus is not new, it recently caught the attention of health officials in the United States. First appearing in New York during the summer of 1999, West Nile killed seven citizens. The symptoms of this disease range from muscle weakness and mental confusion to brain damage and death. For most victims, however, the effects are mild and include headaches, swollen glands, rash, and aching muscles resembling the symptoms that accompany the flu. West Nile encephalitis is spread by birds that have been infected by mosquitoes carrying the virus. The first documentation of infected birds in the U.S. took place in Georgia and the Carolinas. By 2001, as many as twenty states east of the Mississippi River had detected the diseased birds. While less than one percent of mosquitoes carry the virus, those that can live from one summer to the next as a result of warmer winters are the cause for the continuity of the disease from year to year.

During the 1990s, dengue fever appeared in South America and has grown to pandemic proportions with most cases appearing in the Caribbean and India. This viral infection is spread by mosquitoes with symptoms that include severe muscle aches and pain in the bones accompanied by a rash. A typical case lasts for a few weeks. However, dengue hemorrhagic fever is a severe form of the virus that can cause internal bleeding eventually leading to death. Crowded urban areas are more susceptible to outbreaks of dengue fever. In 1993 and 1994, there were epidemics of the disease along the Pacific Coast of Costa Rica. Later during the unusually warm summer of 1995, the range of the disease carrying mosquitoes expanded across the mountains of Costa Rica to invade the rest of the country as well as other parts of Central America and South America. It is estimated that approximately 150,000 people were infected with the virus and thousands of those died due to hemorrhaging. One of the worst outbreaks on record occurred in 2007 throughout the Caribbean affecting hundreds of thousands of inhabitants and killing at least 200 individuals. As these regions are exposed to higher rainfall totals as a result of climate change, more pools of stagnant water along roadside ditches and in open containers contribute to a rise in the number of mosquitoes that carry the virus.

Another relatively new mosquito-borne viral fever is Chikungunya. The symptoms of this viral infection include a sudden high fever, severe headache, and a variety of skin rashes which continue for a week or more. This condition can be accompanied by arthritis-like pain in the bones and joints which persist for weeks or months. The high fever usually lasts a couple of days before finally breaking. Chikungunya and dengue fever are spread by the Aedes albopictus and the Aedes aegypti mosquito, both of which are predominant in the warm, moist regions of the southern United States. According to the Center for Disease Control, approximately 30 cases of Chikungunya have been reported in the U.S. among persons who recently traveled to parts of Asia that had experienced outbreaks of the fever. Chikungunya first appeared in 1952 along the border of Tanganyika and Mozambique in east Africa, and by 1999 it had spread to Malaysia. Thousands of cases were reported in southern India during the fall of 2004, and the virus has since infected more than one million individuals across that country along with another 300,000 on the islands of the Indian Ocean. There is no effective means of curing the virus other than time, and the only preventive treatment is mosquito netting and pesticide. If climate change enhances the monsoonal rains in this region of the world, health officials fear an increase in outbreaks of mosquito-borne diseases which could likely swamp local hospitals and clinics.

The most widespread mosquito-borne disease is malaria. The symptoms consist of headache, weakness, anemia, fever, chills, and intestinal pain. Most victims of malaria survive, but they live with chronic pain. The parasite transmitted by the mosquito may lie dormant in the host and not become active for weeks or months. Once infected, a person may build immunity to the disease. However, in most cases there is the potential of being re-infected multiple times. Additionally, the disease can be transmitted by blood transfusions. There are more cases of malaria today than ever before in human history. The disease has been documented in more than 100 countries. 
Citation: Snow R, Snow M (2015) The Impact of Climate Change on Human Health. J Climatol Weather Forecasting 3: e109. doi:

Page 2 of 2

According to the World Health Organization, between 300 and 500 million people are infected with malaria worldwide. Estimates of the annual average number of deaths from malaria are as a high as 2.5 million with most of these being children who are 14 years of age or younger. The United States reports more than 1000 cases annually. The single-cell parasite causing malaria requires temperatures above $18^{\circ} \mathrm{C}$ in order to develop. The warmer the temperature, the faster the parasites mature. Scientists caution that if global temperatures rise by $2^{\circ} \mathrm{C}$ as forecast, the range of malaria could cover as much as sixty percent of the globe.

There are a host of other diseases commonly associated with warmer temperatures. Cholera appears to be spreading once again as an outbreak occurred in 1991 when the wastewater from an Asian freighter was inadvertently pumped from the hull into the waters off the coast of Peru allowing the cholera organism to thrive in the temperate sea. More than one million cases of cholera were reported as a result along with a staggering 10,000 deaths. After the exceptionally long monsoon season of 1994 in northern India, the temperatures during the subsequent three months were above normal as well as they reached $55^{\circ} \mathrm{C}$ for the daily highs. The hot weather gave rise to an infestation of the fleas that carry pneumonic plague which is transmitted by rats. Hordes of infected rats converged on local Indian communities to escape the surrounding flooded fields carrying with them the dreaded plague which can be transmitted through the air from one person to another. Yellow fever, another mosquito-borne disease which is prevalent in tropical Africa, was confirmed as recently as 2008 in Latin America and has the potential to spread rapidly once it reaches crowded urban areas.

In short, climate change and the associated warming of many regions across the globe will undoubtedly allow many diseases previously confined to the tropics to spread in their range and occurrence. Climate models and scenarios of varying degrees of warming predict a higher number of extreme weather events along with increased precipitation in the middle latitudes where most people live. Since many of the tropical diseases have insect-borne vectors, the warmer and wetter world of the future will undoubtedly have a pronounced impact on human health. 